\title{
Graph Partition by Swendsen-Wang Cuts
}

\author{
Adrian Barbu and Song-Chun Zhu \\ University of California, Los Angeles \\ Departments of Computer Science and Statistics \\ abarbu@ucla.edu,sczhu@stat.ucla.edu
}

\begin{abstract}
Vision tasks, such as segmentation, grouping, recognition, can be formulated as graph partition problems. The recent literature witnessed two popular graph cut algorithms: the Ncut using spectral graph analysis and the minimum-cut using the maximum flow algorithm. This paper presents a third major approach by generalizing the Swendsen-Wang method-a well celebrated algorithm in statistical mechanics. Our algorithm simulates ergodic, reversible Markov chain jumps in the space of graph partitions to sample a posterior probability. At each step, the algorithm splits, merges, or re-groups a sizable subgraph, and achieves fast mixing at low temperature enabling a fast annealing procedure. Experiments show it converges in 230 seconds in a PC for image segmentation. This is 400 times faster than the single-site update Gibbs sampler, and 20-40 times faster than the DDMCMC algorithm. The algorithm can optimize over the number of models and works for general forms of posterior probabilities, so it is more general than the existing graph cut approaches.
\end{abstract}

\section{Introduction}

Computer vision problems, such as image segmentation, perceptual organization, and object recognition, require grouping image elements (pixels, edgelets, primitives) into "coherent" visual patterns (regions, curves, objects) in a process of optimizing some grouping criteria. The problem can be represented in an adjacency graph with the vertices being the image elements, the edges being spatial relationships and subgraphs being coherent visual patterns. Thus it becomes a graph partition problem.

There are two approaches for graph partition in the recent literature. One is the normalized cut $[12,9]$ using graph spectral analysis to optimize a discriminative criterion. The other is the minimum-cut $[8,6]$ which maps an energy minimization problem to a maximum flow algorithm. The latter is solved in polynomial time. Despite their reasonable success, the two approaches are far from being general solu- tions. Firstly it was shown[6] that only very limited classes of energy functions can be mapped to the maximum flow problem. Secondly the graph spectral analysis, like many other discriminative clustering algorithms[3, 2], has difficulties in expressing global visual patterns, such as shading effects, perspective projection effects, contour closure etc. Furthermore natural images contain very diverse visual patterns which are "coherent" in many different ways. This requires a generative and Bayesian formulation incorporating a number of diverse and competing image models[10]. There is no single discriminative criterion that is generally applicable to all the visual patterns.

In this paper, we present a third major graph partition approach by generalizing the Swendsen-Wang method- a well celebrated algorithm in statistical mechanics. Formulated in a Bayesian framework with generative image models, our algorithm simulates ergodic and reversible Markov chain jumps in the space of all possible graph partitions to search for global optima. The basic ideas and contributions of our method are:

1. Given an adjacency graph, we compute a local probability at each edge for how likely the two vertices (image elements) belong to the same pattern. Then by turning on the edges at random according to their associated probabilities, we form connected components, each being a good candidate for a coherent pattern.

2. At each step, the algorithm splits, merges, or regroups a connected component which often includes a big number of vertices. The moves are ergodic and observe detailed balance equations. The candidate states are selected proportional to their posterior probabilities weighted by the probabilities of "graph cuts". The acceptance probability can be made to be always one, and thus our algorithm becomes a generalized Gibbs sampler.

3. The algorithm "mixes" rapidly at low temperature. Unlike most MCMC methods it no longer needs a long simulated annealing procedure[5]. Instead a fast annealing, starting from a lower temperature is used. Thus it can start from good initial conditions using heuristics to achieve a short "burn-in" period. As a result, the algorithm is about 
400 times faster than the classical Gibbs sampler[4] which flips a single vertex each time, and it is 20-40 times faster than the previous DDMCMC algorithm[10]. It converges in $2-30$ seconds in a $1.5 \mathrm{GHz}$ PC for image segmentation.

The central contribution of the paper is the two mathematical theorems for calculating the acceptance probabilities for the big moves, which observe miraculous cancellations in the calculation. The theorems ensure that our algorithm samples from general posterior probabilities, and provide a foundation for fast simulation and optimization for a broad range of vision problems.

\section{Swendsen-Wang: basic ideas}

The difficulty of sampling the graph partition space is well reflected in the Ising and Potts models in statistical mechanics,

$$
p(\mathbf{I}) \propto \exp \left\{\beta \sum_{<s, t>} \mathbf{1}\left(\mathbf{I}_{s}=\mathbf{I}_{t}\right)\right\} \quad \beta>0 .
$$

where $\mathbf{1}\left(\mathbf{I}_{s}=\mathbf{I}_{t}\right)=1$ if $\mathbf{I}_{s}=\mathbf{I}_{t}$ for adjacent spins $s, t$ otherwise it is zero.

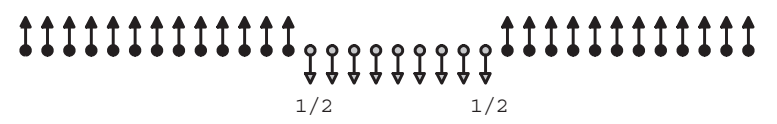

For the string of spins with label $\mathbf{I} \in\{ \pm 1\}^{n}$ shown above, the highest probability is achieved when all vertices have the same label. In a best visiting scheme, the Gibbs sampler flips the -1 spins at the two "cracks" to +1 with probability $p_{o}=1 / 2$. Thus to flip a string of $n$ spins ( $n=9$ here) from -1 to +1 , the expected number of steps is $\frac{1}{\left(1 / p_{o}\right)^{n}}=2^{n}$. This is exponential waiting!

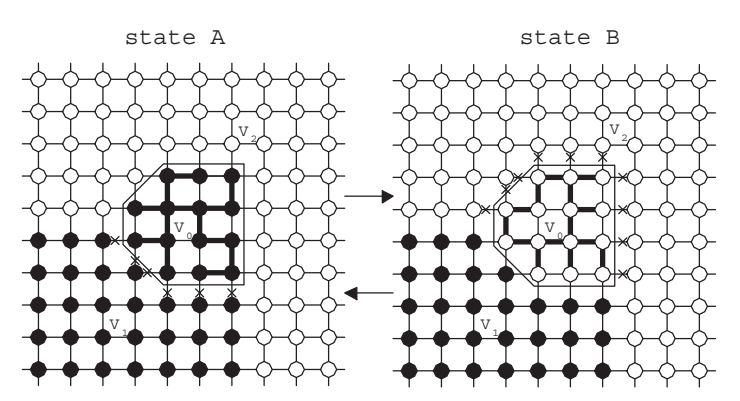

Figure 1. The Swendsen-Wang algorithm flips a patch of spins in one step.

A major speedup for the Ising model in equation (1) is achieved by the Swendsen-Wang (1987) algorithm [11]. E.g. Fig. 1 shows an adjacency graph as a 2D lattice with each edge $e$ connecting two adjacent spins $s, t$. SW turns "on" each edge $e$ with a constant probability $q_{o}=1-e^{-\beta}$ if $s, t$ have the same label. Fig. 1 shows a component $V_{0}$ connected by bold edges which are turned "on" at two states $A$ and $B$. The edges between $V_{0}$ and its neighbors $-V_{1}$ in state $A$ and $V_{2}$ in state $B$ are cut - turned "off", see the crosses in the figure. We denote the two sets of edges by the respective "cuts"

$$
\mathcal{C}\left(V_{0}, V_{1}\right), \quad \mathcal{C}\left(V_{0}, V_{2}\right)
$$

Then SW flips all spins in $V_{0}$ in a single step and makes a reversible jump between states $A$ and $B$. The acceptance probability for the move is shown to be 1 . So SW can flip all -1 spins in the 1D string example in one or a few steps.

The SW algorithm achieves fast mixing even at critical temperature for typical graphs. Unfortunately, it is limited to simple Ising/Potts models and does not use the image (data) information in forming the component $V_{0}$. It is found to be ineffective in the presence of external field (data). In the following, we extend SW to simulating general Bayesian posterior probabilities and make use of bottom-up information to form the candidate components $V_{0}$ to further speed up the computation.

\section{Bayesian formulation of graph partition}

\subsection{Graph Partition}

Let $G_{o}=<V, E_{o}>$ be an adjacency graph where $V=\left\{v_{1}, v_{2}, \ldots, v_{N}\right\}$ is the set of vertices for image elements such as pixels, edgelets, primitives and $E_{o}$ is a set of edges $e=\langle s, t>$ for adjacent elements $s, t$. The objective is to partition graph $G_{o}$ into unknown number of $n$ full subgraphs $G_{k}=<V_{k}, E_{k}>, k=1,2, \ldots, n$, each keeping all the edges in $G_{O}$ that connect its vertices:

$$
\begin{gathered}
V=\cup_{k=1}^{n} V_{k}, \quad V_{k} \neq \emptyset, V_{i} \cap V_{j}=\emptyset \text { for } i \neq j . \\
E_{k}=\left\{e=(u, v) \in E_{o} \mid u, v \in V_{k}\right\}, \quad k=1,2, \ldots, n .
\end{gathered}
$$

We denote by $\pi_{n}$ a partition with $n$ subgraphs.

$$
\pi_{n}=\left\{V_{1}, V_{2}, \ldots, V_{n}\right\} \text { or }\left\{G_{1}, G_{2}, \ldots, G_{n}\right\}
$$

Vertices in each subset $V_{k}, k=1,2 \ldots, n$ forms a coherent visual pattern specified by a generative probability.

The space of all possible partition is denoted by

$$
\Omega_{\pi}=\cup_{n=1}^{|V|} \Omega_{\pi_{n}}
$$

with $\Omega_{\pi_{n}}$ being the space of all $n$-partitions. The edges between any two sets $V_{i}$ and $V_{j}$ are denoted by a $c u t$

$C\left(V_{i}, V_{j}\right)=\left\{e=<s, t>: e \in E_{o}, s \in V_{i}, t \in V_{j}\right\}, \quad i \neq j$.

Fig. 2 shows a typical example of image segmentation. We obtain an over-segmentation (middle) by applying a Canny 


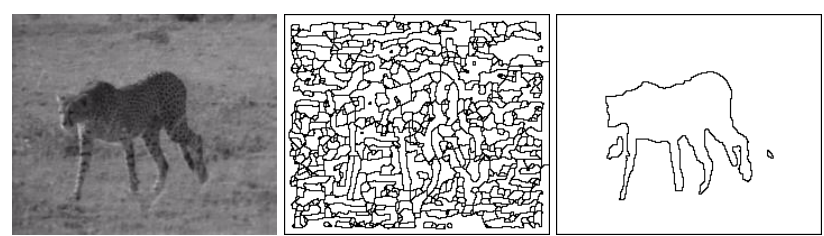

Figure 2. Input image, an over-segmentation with "atomic" regions being vertices, and a segmentation result.

edge detection followed by edge tracing to form "atomic" regions with nearly constant intensities. These atomic regions are the vertices $V_{o}$ and any two adjacent atomic regions are connected by an edge to form the graph $G_{o}$. The right image is a result of our partition algorithm.

\subsection{Solution space and Markov chain design}

Take image segmentation as an example, we denote by $\mathbf{I}_{v}$ the observed image attributes (pixel intensity, edge position and orientation, etc) for element $v$, and by $\mathbf{I}_{V}$ the image representation for the set $V$. Suppose we use $L$ classes of image models for various patterns, such as color, texture, shading, curve etc. Each type of model is indexed by $c \in\left\{C_{1}, C_{2}, \ldots, C_{L}\right\}=\Omega_{C}$, and specified with parameters $\theta_{i} \in \Omega_{c_{i}}$. The model space is the union

$$
\Omega_{\theta}=\cup_{c \in \Omega_{C}} \Omega_{\ell} .
$$

The inner representation for a segmentation is

$$
W=\left(n, \pi_{n},\left(c_{1}, \theta_{1}\right),\left(c_{2}, \theta_{2}\right), \ldots,\left(c_{n}, \theta_{n}\right)\right)
$$

Each subgraph $V_{i}, i=1,2, \ldots, n$ in partition $\pi_{n}$ is specified by a model $p\left(\mathbf{I}_{V_{i}} ; c_{i}, \theta_{c_{i}}\right)$ of type $c_{i}$ and parameters $\theta_{i}$.

The solution space for $W$ is

$$
\Omega=\cup_{n=1}^{N}\left\{\Omega_{\pi_{n}} \times \Omega_{C}^{n} \times \Omega_{c_{1}} \times \cdots \times \Omega_{c_{n}}\right\} .
$$

This factorization of the solution space corresponds to the necessary solution steps:

1. Partition graph $G_{o}$ by finding $\pi_{n} \in \Omega_{\pi}$.

2. Select an image model $c \in \Omega_{C}$ for each subgraph $V_{i} \in \pi_{n}$.

3. Fit the models $\theta_{c_{i}} \in \Omega_{c_{i}}, i=1,2, \ldots, n$.

If we assume the patterns are mutually independent, then the objective is to simulate a Bayesian posterior

$$
W \sim p(W \mid \mathbf{I}) \propto \prod_{i=1}^{n} p\left(\mathbf{I}_{V_{i}} ; c_{i}, \theta_{c_{i}}\right) p(W) .
$$

The prior and image models can be Markov random field models or global spline models, and are beyond what can be minimized by the graph cut algorithms[6,9].
The Markov chain must be ergodic in space $\Omega$ and have $p(W \mid \mathbf{I})$ as its stationary probability. In short, we need two types of reversible jumps[1] bridging the subspaces of different dimensions in $\Omega$.

1. Jumps in the model space $\Omega_{C}^{n} \times \Omega_{c_{1}} \times \cdots \times \Omega_{c_{n}}$, such as model switching, diffusion (fitting) of parameters $\theta_{c}$.

2. Jumps in the partition space $\Omega_{\pi}$ : split, merge, death, birth.

The jumps are realized by Metropolis-Hastings methods[7]. For a pair of states $W=A$ and $W=B$, we need to design proposal probabilities $q(A \rightarrow B)$ and $q(B \rightarrow A)$. The recent idea of data-driven Markov chain Monte Carlo (DDMCMC) in [10] is to calculate them based on bottom-up discriminative models, summarized by $D(\mathbf{I})$, so that the proposal probabilities approximate the posterior

$$
\begin{aligned}
q(B \rightarrow A) & =q(A \mid B, D(\mathbf{I})) \approx p(A \mid \mathbf{I}) \\
q(A \rightarrow B) & =q(B \mid A, D(\mathbf{I})) \approx p(B \mid \mathbf{I})
\end{aligned}
$$

Then the proposed move from $A$ to $B$ is accepted with high probability $\alpha(A \rightarrow B)$

$$
\alpha(A \rightarrow B)=\min \left(1, \frac{q(A \mid B, D(\mathbf{I}))}{q(B \mid A, D(\mathbf{I}))} \cdot \frac{p(B \mid \mathbf{I})}{p(A \mid \mathbf{I})}\right) .
$$

In this paper we use bottom-up data-driven information $D(\mathbf{I})$ and go one step further by making big moves. So the algorithm can reach from a state $A$ to very different $B$ in one step which may need an exponential number of small moves otherwise, as we discussed in the Ising model example.

We follow the DDMCMC method[10] for the jumps in model space, and the rest of the paper is focused on designing smart moves in the partition space $\Omega_{\pi}$ for fast convergence and mixing.

\section{Sampling $\Omega_{\pi}$ with discriminative models}

For an adjacency graph $G_{o}=<V, E_{o}>$, we augment each edge $e=<s, t>\in E_{o}$ with a binary random variable $\mu_{e} \in\{$ on, off $\}$ representing whether the edge is turned "on" or "off". In contrast to a constant probability $q_{o}$ for all edges in the SW algorithm, we compute a discriminative model for $q_{e}=q\left(\mu_{e}=\right.$ on $\left.\mid F(s), F(t)\right)$ based on local vector valued features (texture, color, geometry etc.) $F(s), F(v)$ at the two sites. $q_{e}$ indicates how coherent (or similar) the two vertices $s$ and $t$ are, and can be trained off-line.

By turning "on" each edge $e$ in $G_{o}$ with probability $q_{e}$ independently, we obtain a sparse graph $G=\langle V, E\rangle$ with $E \subset E_{o}$ being the set of edges which are turned on by chance. The probability for $E$ or $G$ is

$$
q(E)=\prod_{e \in E} q_{e} \prod_{e \in E_{o}-E}\left(1-q_{e}\right) .
$$


$G=<V, E>$ consisting of a number $n$ of connected components $g_{k}=<V_{k}, E_{k}>$.

$$
G=\cup_{k=1}^{n} g_{k}, \cup_{k=1}^{n} V_{k}=V, \cup_{k=1}^{n} E_{k}=E .
$$

We denote them by

$$
C P=\left\{V_{1}, V_{2}, \ldots, V_{n}\right\}
$$

As the local probabilities $q_{e}$ are well trained, the subgraphs in $C P$ are often meaningful parts of patterns. This way, $q(E)$ defines a bottom-up probability $q(\pi)$ on the partition space $\Omega_{\pi}$.
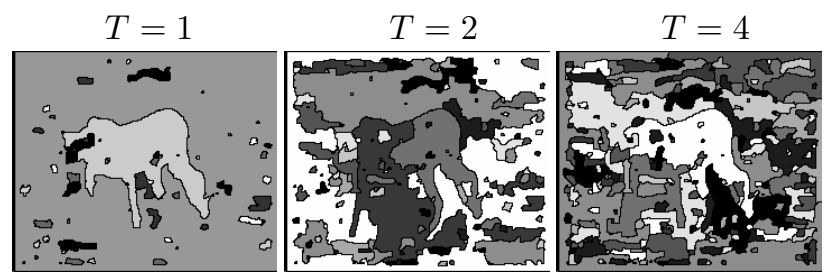

Figure 3. Random samples of $C P$ at $T=1,2,4$ according to $q(E)$.

Figure 3 shows random graph partitions $C P$ for the cheetah image whose adjacency graph $G_{O}$ is built on the atomic regions in Fig. 2 (middle). On each column, we show a $C P$ sampled according to $q(E)$ in equation (5). The size of the components of $C P$ can be controlled by a temperature $T$ on the edge probabilities $q_{e}^{T}$. The smaller the $T$, the larger the size of the components. Clearly various parts of the cheetah are obtained, which will be used as candidates for big and meaningful moves in our MCMC algorithm.

\section{Stochastic graph partition by MCMC}

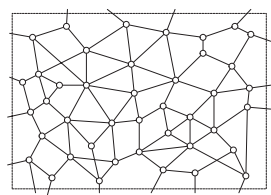

a. $G_{O}$

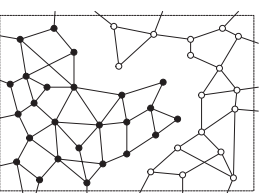

b. $G$

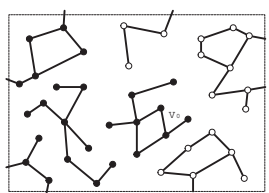

c. $C P$
Figure 4. Three stages of graphs: a. adjacency graph $G_{o}$, b. current partition state $G$, c. a sample from the discriminative models of $G$ and its connected components $C P$.
Our graph partition algorithm operates three graphs shown in Figure 4. It starts with an adjacency graph $G_{o}=<$ $V, E_{o}>$. The current Markov chain state is a partition $\pi: V=\cup_{l=1}^{n} V_{l}$, represented by a graph $G=\cup_{l=1}^{n} G_{l}$, where $G_{l}=<V_{l}, E_{l}>, l=1,2, \ldots, n$ are full subgraphs of $G_{o}$ (Fig.4.b), i.e. $G$ was obtained from $G_{O}$ by removing the edges between the subsets $V_{l}$.

Then during a move between two partition states, it generates connected components $C P$ (Fig.4.c) by turning on/off the edges in $G$. A component in $C P$ is picked up at random as a candidate for reassignment.

Swendsen-Wang Cuts: SWC-1

Input: $G_{o}=<V, E_{o}>$, discriminative probabilities $q_{e}, \forall e \in E_{o}$, and generative posterior probability $p(W \mid \mathbf{I})$.

Output: Samples $W \sim p(W \mid \mathbf{I})$.

1. Initialize a graph partition $\pi: G=\cup_{l=1}^{n} G_{l}$.

2. Repeat, for current state A

3. Repeat for each subgraph $G_{l}=<V_{l}, E_{l}>, l=1,2, \ldots, n$

4. For $e \in E_{l}$, turn $\mu_{e}=$ on with probability $q_{e}$.

5. $V_{l}$ is divided into $n_{l}$ connected components: $\left\{g_{l i}=<V_{l i}, E_{l i}>, i=1, \ldots, n_{l}\right\}$.

6. Collect connected components from all subgraphs (see Fig.4.c) $C P=\left\{V_{l i}: l=1, \ldots, n, i=1, \ldots, n_{l}\right\}$.

7. Select a component $V_{0} \in C P$ at random with probability $q\left(V_{0} \mid C P\right)$, (usually $1 /|C P|$ ) (see Fig.5.a).

8. Propose to assign $V_{0}$ to a subgraph $G_{l^{\prime}} . l^{\prime}$ follows a probability $q\left(l^{\prime} \mid V_{0}, A, G_{o}\right)$ (state $B$ in Fig.5.b if $V_{0}$ is merged to an existing subgraph, state $C$ in Fig.5.c if $V_{0}$ is a new subgraph).

9. Accept the move with probability $\alpha(A \rightarrow B)$ or $\alpha(A \rightarrow C)$ in theorem 1.

We omit the parallel steps of model switching and fitting for clarity. The probability $q\left(l^{\prime} \mid V_{0}, A, G_{o}\right), l^{\prime}=1, \ldots, n+1$ can be designed simply as follows:

$$
q\left(l^{\prime} \mid V_{0}, A, G_{o}\right)= \begin{cases}a & \text { if } G_{l^{\prime}} \text { is adjacent to } \mathrm{V}_{0}, \\ b & \text { if } l^{\prime}=n+1, \text { new subgraph } \\ c & \text { else }\end{cases}
$$
$10 c$

such that $\sum_{l^{\prime}=1}^{n+1} q\left(l^{\prime} \mid V_{0}, A, G_{o}\right)=1$. Usually $a=b=$

The move between states $A$ and $B$ is a split-merge operation in canonical cases. Two special cases are the birth and death moves.

1. If $l^{\prime}=n+1, V_{0}$ becomes a new subgraph, so the move is a birth operation.

2. If $V_{0}$ is equal to a subgraph $V_{l}$, the whole subgraph $G_{l}$ is merged to $G_{l^{\prime}}$. The number of subgraphs is reduced by one, so it is a death operation.

In what follows, we give a simple, explicit expression for the acceptance probability, which can be made to be 1 through a smarter choice of $q\left(l^{\prime} \mid V_{0}, A, G_{o}\right)$.

Theorem 1 In the above notation, consider a candidate component $V_{0}$ selected by $\mathrm{SWC}-1$. If the proposed move to reassign $V_{0}$ from $G_{l}$ to $G_{l^{\prime}}$ is accepted with probability

$$
\alpha(A \rightarrow B)=\min \left(1, \frac{\prod_{e \in \mathcal{C}\left(V_{0}, V_{l^{\prime}}-V_{0}\right)}\left(1-q_{e}\right)}{\prod_{e \in \mathcal{C}\left(V_{0}, V_{l}-V_{0}\right)}\left(1-q_{e}\right)} \frac{q\left(l \mid V_{0}, B, G_{o}\right)}{q\left(l^{\prime} \mid V_{0}, A, G_{o}\right)} \frac{p(B \mid \mathbf{I})}{p(A \mid \mathbf{I})}\right)
$$


then the Markov chain is ergodic and observes the detailed balance equations.

In the special case when $l^{\prime}=n+1, V_{0}$ is proposed to be a new subgraph and $V_{l^{\prime}}-V_{0}=\emptyset$. So the cut is empty $\mathcal{C}\left(V_{0}, V_{l^{\prime}}-V_{0}\right)=\emptyset, \prod_{e \in \mathcal{C}\left(V_{0}, V_{l^{\prime}}-V_{0}\right)}\left(1-q_{e}\right)=1$ and $\alpha(A \rightarrow B)$ becomes $\alpha(A \rightarrow C)$.

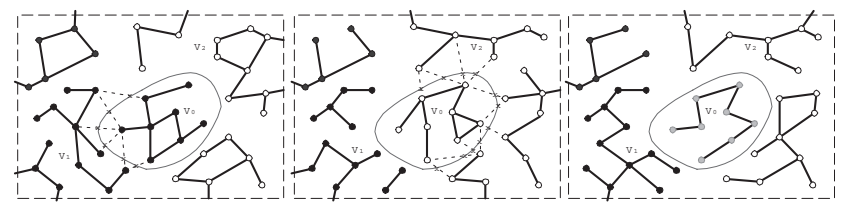
a. state A
b. state B
c. state $\mathrm{C}$

Figure 5. A move between partition states $\pi=A, B, C$, different by a set of vertices $V_{0}$. The vertices in the same color belong to a subgraph. The vertices connected by thick edges form a connected component.

Proof. The idea of the proof is that even though the proposal probabilities $q(A \rightarrow B)$ and $q(B \rightarrow A)$ are very complicated, their ratio $q(B \rightarrow A) / q(A \rightarrow B)$ is extremely simple through miraculous cancellation. Then the conclusion follows from the Metropolis-Hastings equation (4).

First, we calculate the proposal probability $q(A \rightarrow B)$ in SWC-1, assuming state $A$ has $n$ subgraphs $G_{l}=\left\langle V_{l}, E_{l}\right\rangle$ $, l=1,2, \ldots, n$. In the canonical case when $V_{0} \neq V_{l}$ and $V_{l^{\prime}} \neq \emptyset$, it is a conditional probability which consists of two steps: (1) choosing $V_{0}$ and (2) choosing $l^{\prime}$. For clarity, we discuss the exception cases later.

In state A, each subgraph $G_{l}$ is broken into connected components $C P_{l}$ by turning on and off the edges in $E_{l}$ at random. We denote the set of all connected components

$$
C P(A)=\cup_{l} C P_{l}=\left\{V_{l i}: l=1, \ldots, n ; i=1, \ldots, n_{l}\right\} .
$$

For example, Figure 5.a shows 6 connected components. For a $C P$ of state $A$, we denote by $E_{\text {on }}(A, C P)$ the edges that are turned on (the thick edges in Figure 5.a)

$$
E_{\text {on }}(A, C P)=\cup_{l=1}^{n}\left\{\cup_{i=1}^{n_{l}} E_{k i}\right\} .
$$

The rest of the edges, which are turned off, are the "cuts" between a connected component $V_{l i}$ and other vertices in the subgraph, i.e. $V_{l}-V_{l i}$,

$E_{\text {off }}(A, C P)=\cup_{l=1}^{n}\left\{\cup_{i=1}^{n_{l}} C_{l i}\right\}, \quad C_{l i}=C\left(V_{l i}, V_{l}-V_{l i}\right)$.

Note that the edges between subgraphs had been turned off before entering state $A$. The probability for choosing a $C P$ depends on state $A$ and the discriminative models $D(\mathbf{I})$,

$$
q(C P \mid A, D(\mathbf{I}))=\prod_{e \in E_{\mathrm{on}}(A, C P)} q_{e} \prod_{e \in E_{\mathrm{off}}(A, C P)}\left(1-q_{e}\right) .
$$

We denote by $\Omega_{C P}(A)$ the set of all possible $C P$ 's at state $A$. We are interested in those $C P$ 's which contain $V_{0}$,

$$
\Omega_{C P}^{0}(A)=\left\{C P(A): V_{0} \in C P\right\} .
$$

Without loss of generality, we assume that $V_{0}$ is a component from subgraph $G_{1}=<V_{1}, E_{1}>$. We denote the cut between $V_{0}$ and $V_{1}-V_{0}$ by $\mathcal{C}_{01}=C\left(V_{0}, V_{1}-V_{0}\right)$.

All $C P \mathrm{~s}$ in $\Omega_{C P}^{0}(A)$ have the following two properties: they all contain $V_{0}$, and all edges between $V_{0}$ and $V_{1}-V_{0}$ are turned off (otherwise $V_{0}$ is connected to other vertices). In other words, $\forall C P \in \Omega_{C P}^{0}(A)$

$$
V_{0} \in C P \text { and } C_{01} \subset E_{\text {off }}(A, C P) .
$$

For each $C P \in \Omega_{C P}^{0}(A)$, the set $V_{0}$ is picked with a probability $q\left(V_{0} \mid C P\right)$. Now we are ready to compute the probability for selecting $V_{0}$ at state $A$,

$$
\begin{aligned}
& q\left(V_{0} \mid A, D(\mathbf{I})\right)=\sum_{C P \in \Omega_{C P}^{0}(A)} q\left(V_{0} \mid C P\right) q(C P \mid A, D(\mathbf{I})) \\
= & \prod_{e \in \mathcal{C}_{01}}\left(1-q_{e}\right)\left[\sum_{C P \in \Omega_{C P}^{0}(A)} q\left(V_{0} \mid C P\right) \prod_{e \in E_{\text {off }}(A, C P)-\mathcal{C}_{01}}\left(1-q_{e}\right) \prod_{e \in E_{\text {on }}(A, C P)} q_{e}\right] .
\end{aligned}
$$

We were able to factor the product $\prod_{e \in C_{01}}\left(1-q_{e}\right)$ out because $C_{01} \subset E_{\text {off }}(A, C P)$ for all $C P \in \Omega_{C P}^{0}(A)$.

Once $V_{0}$ is selected, it is assigned to $G_{l^{\prime}}$ with probability $q\left(l^{\prime} \mid V_{0}, A, G_{o}\right)$, the same for all $C P \in \Omega_{C P}^{0}(A)$. Therefore, the proposal probability from $A$ to $B$ is,

$$
q(A \rightarrow B)=q\left(V_{0} \mid A, D(\mathbf{I})\right) q\left(l^{\prime} \mid V_{0}, A, G_{o}\right) .
$$

Now we calculate the proposal probability $q(B \rightarrow A)$ in algorithm SWC-1. In the canonical case, the only way one can get from state $B$ to state $A$ is by selecting $V_{0}$ as a connected component and re-assigning it to $G_{l}$.

In state $B$, we have the same partition as in state $A$ except that $V_{0}$ belongs to $G_{l^{\prime}}$ (see Fig. 5.b). Without loss of generality, we assume that $V_{0}$ is a component from the subgraph $G_{2}=<V_{2}, E_{2}>. \Omega_{C P}^{0}(B)$ is the set of $C P$ 's that contain $V_{0}$ as a component and must share the common cut $\mathcal{C}_{02}=C\left(V_{0}, V_{2}-V_{0}\right)$, illustrated in Figure 5 .b by the crosses. Similarly, the probability for selecting $V_{0}$ at state $B$ is,

$$
\begin{aligned}
& q\left(V_{0} \mid B, D(\mathbf{I})\right)=\sum_{C P \in \Omega_{C P}^{0}(B)} q\left(V_{0} \mid C P\right) q(C P \mid B, D(\mathbf{I}))(10) \\
= & \prod_{e \in \mathcal{C}_{02}}\left(1-q_{e}\right)\left[\sum_{C P \in \Omega_{C P}^{0}(B)} q\left(V_{0} \mid C P\right) \prod_{e \in E_{\text {off }}(B, C P)-\mathcal{C}_{02}}\left(1-q_{e}\right) \prod_{e \in E_{\mathrm{on}}(B, C P)} q_{e}\right],
\end{aligned}
$$

and the proposal probability from $B$ to $A$ is,

$$
q(B \rightarrow A)=q\left(V_{0} \mid B, D(\mathbf{I})\right) q\left(l \mid V_{0}, B, G_{o}\right) .
$$


Observation. For each $C P \in \Omega_{C P}^{0}(A)$, then $C P \in$ $\Omega_{C P}^{0}(B)$ and vice versa. Therefore we have

$$
\Omega_{C P}^{0}(A)=\Omega_{C P}^{0}(B)
$$

For any $C P$ above, the set of edges turned on are the same,

$$
E_{\text {on }}(A, C P)=E_{\text {on }}(B, C P)
$$

and the set of edges turned off are also the same except cut $C_{01}$ occurs in state $A$ and cut $C_{02}$ occurs in state $B$. So

$$
E_{\text {off }}(A, C P)-\mathcal{C}_{01}=E_{\text {off }}(B, C P)-\mathcal{C}_{02} .
$$

Plug in equations (13) and (14) into equations (9) and (11), we have the probability ratio by cancellation,

$$
\frac{q\left(V_{0} \mid B, D(\mathbf{I})\right)}{q\left(V_{0} \mid A, D(\mathbf{I})\right)}=\frac{\prod_{e \in \mathcal{C}_{02}}\left(1-q_{e}\right)}{\prod_{e \in \mathcal{C}_{01}}\left(1-q_{e}\right)}
$$

Therefore,

$$
\frac{q(B \rightarrow A)}{q(A \rightarrow B)}=\frac{\prod_{e \in \mathcal{C}_{02}}\left(1-q_{e}\right)}{\prod_{e \in \mathcal{C}_{01}}\left(1-q_{e}\right)} \cdot \frac{q\left(l \mid V_{0}, B, G_{o}\right)}{q\left(l^{\prime} \mid V_{0}, A, G_{o}\right)} .
$$

By equation (4), we obtain $\alpha(A \rightarrow B)$ as the theorem states. Thus the move between $A$ and $B$ observes the detailed balance equations.

The above proof is for the canonical case when there is only one way to go from state A to state B, or from state B to state A, namely by reassigning $V_{0}$.
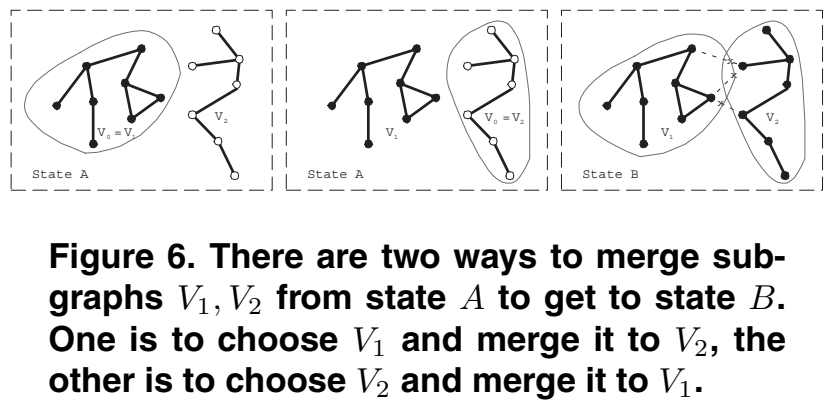

There is an exception to the canonical case when there are two paths between states $A$ and $B$. It occurs when a whole subgraph $G_{l}$ or $G_{l^{\prime}}$ is chosen as $V_{0}$ in state $A$, and thus two subgraphs are merged in state $B$. Without loss of generality, we only consider two subgraphs $V_{1}, V_{2}$ in state $A$ and one subgraph $V_{1} \cup V_{2}$ in state $B$, as Fig. 6 displays.

- Path 1. Choose $V_{0}=V_{1}$. In state $A$, choose $l^{\prime}=2$, i.e. merge it to $V_{2}$, and reversely in state $B$, choose $l^{\prime}=1$, i.e. split it from $V_{2}$.

- Path 2. Choose $V_{0}=V_{2}$. In state $A$, choose $l^{\prime}=1$, i.e. merge it to $V_{1}$, and reversely in state $B$, choose $l^{\prime}=2$, i.e. split it from $V_{1}$.
Thus the proposal probability $q(A \rightarrow B)$ is the sum of the probabilities for the two paths.

$$
\begin{aligned}
q(A \rightarrow B) & =q\left(l^{\prime}=2 \mid V_{1}, A, G_{o}\right) q\left(V_{1} \mid A, D(\mathbf{I})\right) \\
& +q\left(l^{\prime}=1 \mid V_{2}, A, G_{o}\right) q\left(V_{2} \mid A, D(\mathbf{I})\right)
\end{aligned}
$$

and similarly

$$
\begin{aligned}
q(B \rightarrow A) & =q\left(l^{\prime}=1 \mid V_{1}, B, G_{o}\right) q\left(V_{0}=V_{1} \mid B, D(\mathbf{I})\right) \\
& +q\left(l^{\prime}=2 \mid V_{2}, B, G_{o}\right) q\left(V_{0}=V_{2} \mid B, D(\mathbf{I})\right) .
\end{aligned}
$$

In state $A$, the cut is $\mathcal{C}\left(V_{0}, V_{l}-V_{0}\right)=\mathcal{C}\left(V_{0}, \emptyset\right)=\emptyset$ for both paths, and in state $B$ the cut is $\mathcal{C}\left(V_{0}, V_{l}-V_{0}\right)=\mathcal{C}\left(V_{1}, V_{2}\right)=$ $\mathcal{C}_{12}$ for both paths.

Following previous calculation, we have the proposal probability ratio for choosing $V_{0}=V_{1}$ in path 1 ,

$\frac{q\left(V_{0}=V_{1} \mid B, D(\mathbf{I})\right)}{q\left(V_{0}=V_{1} \mid A, D(\mathbf{I})\right)}=\frac{\prod_{e \in \mathcal{C}\left(V_{1}, V_{2}\right)}\left(1-q_{e}\right)}{\prod_{e \in \mathcal{C}\left(V_{1}, \emptyset\right)}\left(1-q_{e}\right)}=\prod_{e \in \mathcal{C}_{12}}\left(1-q_{e}\right)$.

Similarly, we have the probability ratio for choosing $V_{0}=$ $V_{2}$ in path 2 ,

$\frac{q\left(V_{0}=V_{2} \mid B, D(\mathbf{I})\right)}{q\left(V_{0}=V_{2} \mid A, D(\mathbf{I})\right)}=\frac{\prod_{e \in \mathcal{C}\left(V_{2}, V_{1}\right)}\left(1-q_{e}\right)}{\prod_{e \in \mathcal{C}\left(V_{2}, \emptyset\right)}\left(1-q_{e}\right)}=\prod_{e \in \mathcal{C}_{12}}\left(1-q_{e}\right)$.

Plug in the above equations, we obtain the ratio,

$$
\frac{q(B \rightarrow A)}{q(A \rightarrow B)}=\prod_{e \in \mathcal{C}_{12}}\left(1-q_{e}\right)
$$

.$\frac{q\left(l^{\prime}=1 \mid V_{1}, B, G_{o}\right) q\left(V_{1} \mid A, D(\mathbf{I})\right)+q\left(l^{\prime}=2 \mid V_{2}, B, G_{o}\right) q\left(V_{2} \mid A, D(\mathbf{I})\right)}{q\left(l^{\prime}=2 \mid V_{1}, A, G_{o}\right) q\left(V_{1} \mid A, D(\mathbf{I})\right)+q\left(l^{\prime}=1 \mid V_{2}, A, G_{o}\right) q\left(V_{2} \mid A, D(\mathbf{I})\right)}$

The proposal probabilities for $l^{\prime}$ must be designed in such a way that:

$$
\frac{q\left(l^{\prime}=1 \mid V_{1}, B, G_{o}\right)}{q\left(l^{\prime}=2 \mid V_{1}, A, G_{o}\right)}=\frac{q\left(l^{\prime}=2 \mid V_{2}, B, G_{o}\right)}{q\left(l^{\prime}=1 \mid V_{2}, A, G_{o}\right)}
$$

This is easily satisfied in general. So we have,

$$
\frac{q(B \rightarrow A)}{q(A \rightarrow B)}=\prod_{e \in \mathcal{C}\left(V_{0}, V_{l^{\prime}}-V_{0}\right)}\left(1-q_{e}\right) \cdot \frac{q\left(l^{\prime}=1 \mid V_{1}, B, G_{o}\right)}{q\left(l^{\prime}=2 \mid V_{1}, A, G_{o}\right)}
$$

In general notation, it is

$$
\frac{q(B \rightarrow A)}{q(A \rightarrow B)}=\frac{\prod_{e \in \mathcal{C}\left(V_{0}, V_{l^{\prime}}-V_{0}\right)}\left(1-q_{e}\right)}{\prod_{e \in \mathcal{C}\left(V_{0}, V_{l}-V_{0}\right)}\left(1-q_{e}\right)} \cdot \frac{q\left(l \mid V_{0}, B, G_{o}\right)}{q\left(l^{\prime} \mid V_{0}, A, G_{o}\right)}
$$

Thus we have proved the exception case.

To prove ergodicity, observe that there is a non-zero probability that any given node is chosen as a connected component $V_{0}$. Since the node can then be assigned to any other subgraph with nonzero probability, and this holds for all nodes independently, we can get from any partition to any other partition with non-zero probability. End of Proof. 
Now we shall construct $q\left(l^{\prime} \mid V_{0}, A, G_{o}\right)$ in such a way to obtain acceptance probability 1 . Then our algorithm becomes a generalized Gibbs sampler.

Suppose the Markov chain is at a partition state $A=$ $\left(V_{1}, V_{2}, \ldots, V_{n}\right)$, and a connected component $V_{0} \subset V_{l}$ is selected by SWC-1 as a candidate set. We have $n+1$ choices for state $B$ by assigning $V_{0}$ to one of the following vertex sets:

$\left\{S_{1}=V_{1}, S_{2}=V_{2}, \ldots, S_{l}=V_{l}-V_{0}, \ldots, S_{n}=V_{n}, S_{n+1}=\emptyset\right\}$

We denote the states as $B_{1}, B_{2}, \ldots, B_{n+1}$ respectively. Clearly $B_{l}=A$ and in state $B_{n+1}, V_{0}$ is a new subgraph. In the exception case $V_{0}=V_{l}$, then the state $B_{n+1}=A$ is redundant, so one of them should be eliminated.

Denote the cuts between $V_{0}$ and $S_{j}$ by $\mathcal{C}_{j}=C\left(V_{0}, S_{j}\right)$ $j=1,2, \ldots, n+1$ with $\mathcal{C}\left(V_{0}, \emptyset\right)=\emptyset$.

Theorem 2 In the above notation, suppose $V_{0}$ is a candidate vertex set selected by $S W C-1$, in partition state A. If the probabilities for merging $V_{0}$ to $V_{l^{\prime}}$ are chosen to be

$$
q\left(l^{\prime} \mid V_{0}, A, G_{o}\right) \propto \prod_{e \in \mathcal{C}_{l^{\prime}}}\left(1-q_{e}\right) \cdot p\left(B_{l^{\prime}} \mid \mathbf{I}\right) .
$$

then the proposed move is accepted with probability $\alpha\left(A \rightarrow B_{l^{\prime}}\right)=1$.

The proof is straightforward and we omit it. We also omit the proof that $q\left(l^{\prime} \mid V_{0}, A, G_{o}\right)$ satisfies condition (21). In practice, the posteriors $p(A \mid \mathbf{I})$ and $p\left(B_{l^{\prime}} \mid \mathbf{I}\right)$ only involve local computation and the cuts $\mathcal{C}_{l^{\prime}}$ are small or empty.

Intuitively, our algorithm samples a random set of vertices according to posterior for goodness-of-fit modulated by the cut probability to achieve detailed balance. This is much more general than the original SW-method [11] and the Gibbs sampler [4].

\section{Experiments and performance analysis}

The image segmentation experiment was performed on "atomic regions" obtained by edge detection and edge tracing. They form the nodes of our graph. The discriminative probability $q_{e}$ for an edge $e=<v_{i}, v_{j}>$ is

$$
q_{e}=0.1+0.9 e^{-\left(K L\left(p_{i} \| p_{j}\right)+K L\left(p_{j} \| p_{i}\right)\right) / 2} .
$$

where $p_{i}, p_{j}$ are 15 bin intensity histograms in the atomic regions, and $K L()$ is the Kullback-Leibler divergence. In general, this $q_{e}$ can be learned through supervised learning. We use three simple image models $\left\{C_{1}, C_{2}, C_{3}\right\}$ (constant, linear and quadratic polynomial intensity) with additive noise modeled by a 15 bin histogram $\mathcal{H}$.

In Fig. 8 we plotted the energy vs time (in seconds) of 5 runs of the Swendsen-Wang Cuts (SWC-1) algorithm and

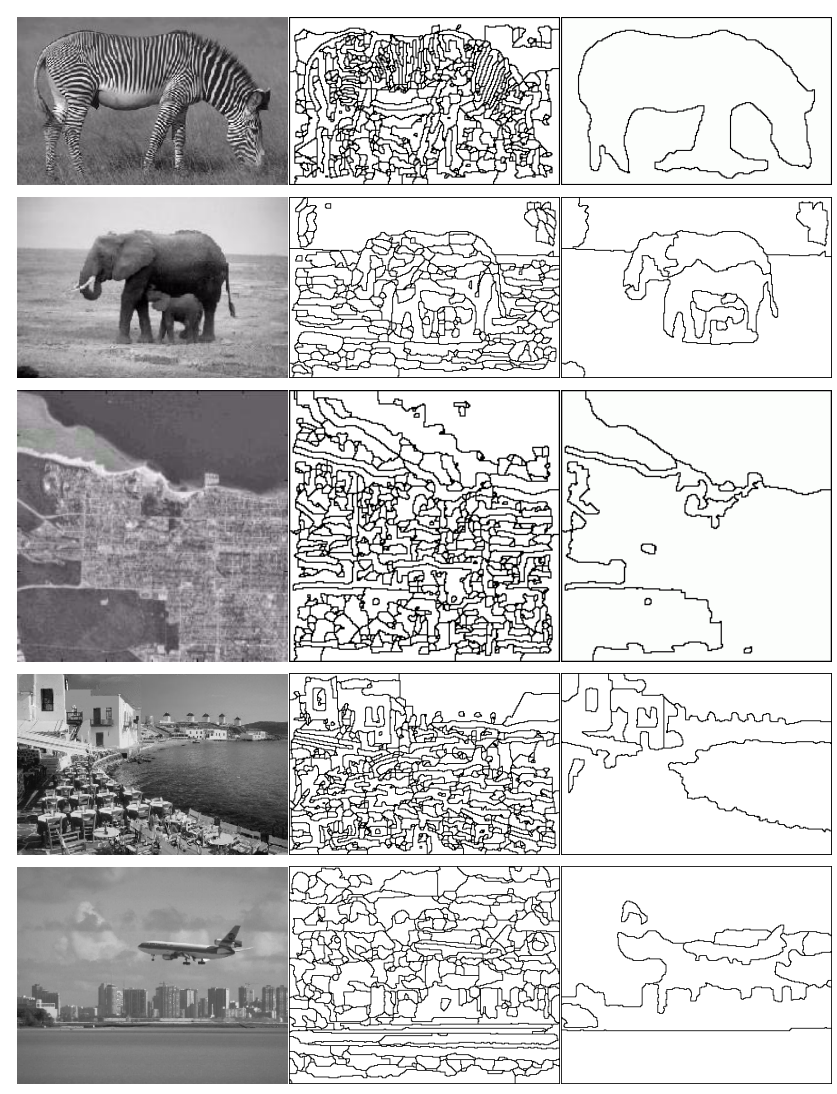

Figure 7. Image segmentation: input image, atomic regions as image elements and the segmentation result.
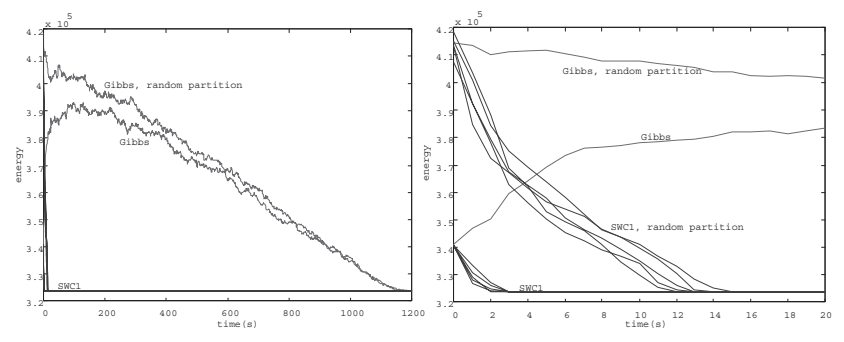

Figure 8. Convergence comparison with Gibbs sampler (upper curves) for the cheetah image. The Gibbs sampler must start with a high temperature and anneal slowly to get to the minimum energy level of our algorithm. Right plot shows a zoom-in view of the first 20 seconds.

one run of the Gibbs sampler on the cheetah image in Fig.2, starting from random partition and 5 runs starting from $\pi=$ 
$\left\{G_{o}\right\}$ (partition with one subgraph). The Gibbs samplers converges in about 1200 seconds and our algorithm in $15 \mathrm{~s}$ if starting from random partition, and 3 seconds if starting from a one subgraph partition. The convergence is faster in latter case since the algorithm started from a lower initial energy. Observe that our algorithm converges 400 times faster than the Gibbs sampler.
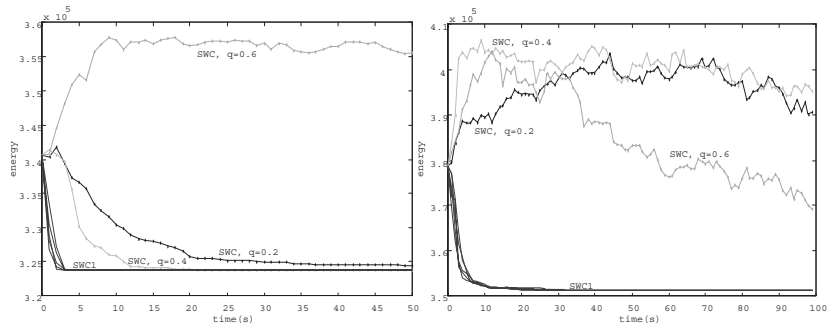

Figure 9. Convergence comparison with SWC without discriminative models $\left(q_{e}=\right.$ $0.2,0.4,0.6)$ (dotted curves). Cheetah image (left), airplane image (right). The original Swendsen-Wang does not apply in this case.

In Fig.9 we made a comparison of our algorithm with and without the discriminative models, on the cheetah image (left) and airplane image (right), starting from $\pi=$ $\left\{G_{o}\right\}$. For that, we plotted the energy vs time of 5 runs of our SWC-1 algorithm with discriminative models (smooth curves) and without discriminative models (dotted curves), where we fixed the edge weights to constants $q_{e}=0.2,0.4,0.6$. The convergence slows down significantly, the annealing schedule must be much slower and the initial temperature higher without the discriminative models.

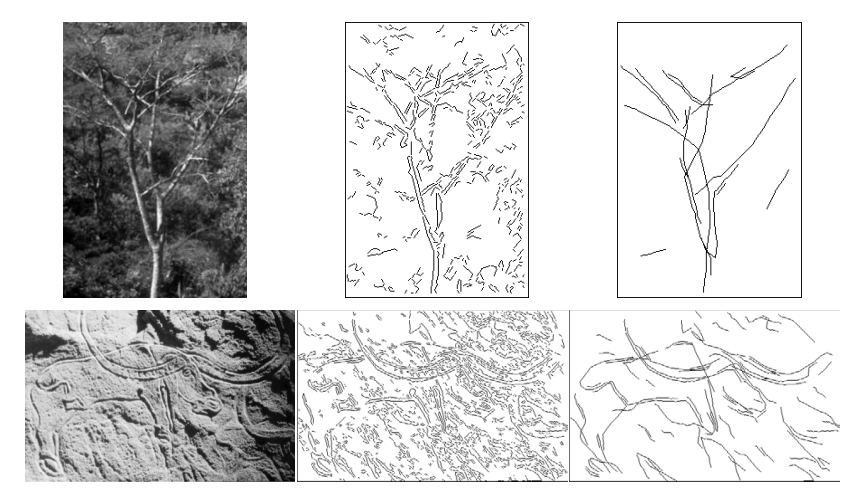

Figure 10. The curve grouping experiment: input image, edge map and grouping result.
In the perceptual grouping experiment we group a map of edgelets obtained from a Canny edge map into long and smooth curves by adding and removing edgelets. The curve prior is based on 3 point histograms learned from hand segmented examples. The likelihood measures the difference in pixels between the input edge map and the grouping result. The graph nodes are the edgelets, and the discriminative probability $q_{e}$ is based on the 3-point histogram and on the gap that has to be filled between the edgelets. The results are shown in Fig.10.

\section{References}

[1] P. J. Green, "Reversible jump MCMC comput. and Bayes. model determination",Biometrika,82, 711-32 1995

[2] T. Hofmann, J.M. Buhmann, "Pairwise data clustering by deterministic annealing”, PAMI, 19(1), 1-14, 1997.

[3] A.K. Jain, R. Dubes, Algorithms for Clustering Data, Prentice Hall, Englewood Cliffs, NJ, 1988.

[4] S. Geman, D. Geman, "Stochastic relaxation, Gibbs distrib., Bayesian restoration of img", PAMI, vol. 6, 721-741, 1984.

[5] S. Kirkpatrick, C. Gelatt, M. Vecchi, "Optimization by simulated annealing”, Science, 220(4598), 671-680, 1983.

[6] V. Kolmogorov, R. Zabih, "What energy functions can be min. via graph cuts?", $E C C V, 2002$

[7] N. Metropolis, et al. "Eqns of the state calculation by fast computing machines", J. Chem. Phys, 21, 1087-91, 1953.

[8] S. Roy, I. Cox, "A max-flow formulation of the n-camera stereo correspondence problem”, ICCV, 1998.

[9] J. Shi, J. Malik, "Normalized cuts and image segmentation", PAMI, 22, no 8, pp. 888-905, 2000.

[10] Z.W. Tu, S. C. Zhu, "Image segmentation by data-driven MCMC”, PAMI, 24, no. 5, 2002.

[11] R.H. Swendsen, J.S. Wang, "Nonuniversal critical dynamics in MC simulations", Phys. Rev. Lett., 58 no. 2, pp.86-88, 1987

[12] Z. Wu and R. Leahy, "An optimal graph theoretic approach to data clustering PAMI, vol.15, 1101-1113, 1993. 